Hospital (RSCH) has developed a Personalised End of Life Care Plan (PELICan). This aim of this project is to assess the use of PELICans in a District General Hospital and Cancer Centre over a three year period.

Methods A database was created of all patients with a PELICan at the RSCH over a three year time period (1/9/2014$31 / 8 / 2017)$. Outcome measures included start date of PELICan, presence of PELICan over a weekend and length of time on PELICan before death or discontinuation.

Results 1378 patients had a PELICan over three years. There was an increasing number of patients placed with a PELICan every year $(53 \%, 55 \%$ and $62 \%$ in successive years). The mean time a patient was cared for with a PELICan before death or discontinuation was 4.5 days (range 1-169 days). Only $7 \%$ of PELICans were discontinued over three years, with $71.4 \%$ being stopped in the first seven days. $16.8 \%$ percent of PELiCans were commenced over the weekend, with this number increasing every year $(14.5 \%, 16.0 \%$ and $19.6 \%$ in successive years). Of those patients who died with a PELICan, $27.2 \%$ died on a weekend.

Conclusion The use of the PELICan is increasing at the $\mathrm{RSCH}$. There has been the introduction of a seven day Palliative care service at the $\mathrm{RSCH}$, which is imperative when considering the proportion of patients dying with a PELICan, as well as those dying on a weekend. Physicians are quite accurate with their prediction of death within the next seven days. The PELICan booklet at the $\mathrm{RSCH}$ requires a daily multidisciplinary review, and consequently, most of the PELICans that are discontinued are done so early on.

\section{QUALITY OF DISCHARGE SUMMARIES FOR PATIENTS SENT HOME FROM HOSPITAL FOR END OF LIFE CARE - A REPEAT AUDIT}

Anirudh Kumar, Sophie Hunter, Ruth England. University Hospitals of Derby and Burton NHS Trust

\subsection{6/bmjspcare-2019-ASP.61}

Background $30 \%$ of patients leaving acute hospitals die within 12 months. When patients leave hospital, the discharge summary letter must give adequate, appropriate information to primary care providers so they can optimise ongoing care. The standard of discharge summaries is variable; evidence suggests that quality improvement projects can enhance their value.

Methods This single centre, retrospective, repeat audit carried out by F2 doctors across an acute hospital examined the quality of 58 discharge letters written between 1 st August and 31st October 2017 for patients discharged home with 'fast-track' support, anticipated to be at the end of their life. Written information in discharge summaries was audited against standards from the Royal College of Physicians as well as supplementary local standards developed for use when patients are believed to be in the last weeks of their life.

Results There is still room to improve the quality of discharge summaries for patients being 'fast-tracked' home. Compared with a baseline audit in 2015, more patients were prescribed anticipatory medications on discharge and more 'sensitive discussions' were documented. However, fewer summaries contained documentation of a patient's preference for place of care and fewer had instructions to GPs regarding care at the end of life. There was no documentation about provision of patient information leaflets.

Conclusions Engaging junior doctors in a repeat audit cycle has an impact on quality of discharge summaries. Stickers to prompt content of discharge summaries have been developed and refined. Additional teaching has been provided to Foundation Year doctors. An information leaflet for patients explaining the fast-track process has been created. A further re-audit is planned in 2019 to determine the impact of these interventions.

\section{ARE WE MAKING THINGS ANY BETTER? ASSESSING THE QUALITY OF A PERSONALISED CARE PLAN FOR THE LAST DAYS OF LIFE WITHIN AN ACUTE HOSPITAL TRUST}

J Winter, B Francis, H McDonald, F Malik. East Sussex Healthcare NHS Trust

\subsection{6/bmjspcare-2019-ASP.62}

Background Following CQC inspection, need for improvement in recognising dying/individualised care planning in one acute NHS trust was highlighted. In response, the Last Days of Life Personalised Care Plan (LDoLPCP) was developed based on NICE guidance and Five Priorities for care of dying person. Implementation audited via multiple methods.

Methods

- Retrospective cross-sectional monthly spot audits Nov 17-Apr 18 in two acute hospitals. Those on LDOLCP identified from SPCT current case-list, ward visits \& mortality database/ bereavement office data. 10 care plans reviewed monthly each site. Two clinicians assessed quality of documentation using a standardised audit tool. Areas covered include: recognition, senior decision maker, anticipatory prescribing etc. Each section subjectively rated (High standard, acceptable, needs improvement, poor). Data collated anonymously. Results analysed using summary statistics. Target level for standards set at $80 \%$ of plans completed to a high/acceptable level.

- Online survey questionnaire emailed to all Trust frontline staff to ascertain views/feedback on LDOLPCP. Qualitative feedback gained from frontline Nursing staff on 20 wards cross-site.

\section{Results}

- 110 care plans reviewed. Mean age 82 years, 56\% female. In $73 \%(80 / 110)$, section on recognition of dying completed to high or acceptable level. Highest scores: $83 \%$ for documentation of senior doctor \& 92.9\% for anticipatory prescribing for pain $\&$ nausea. However in other areas, results fell below target including Emotional/spiritual/cultural assessment $36.7 \%$; daily symptom assessment $43.0 \%$ and Hydration status assessed, $48.5 \%$. Reasons for poor quality documentation include uncertainty over whom best to complete, time pressures, LDOLPCP viewed as one-off document rather than an evolving plan.

- Online survey in progress \& reaudit - results to follow.

Conclusion Audit shows areas of mixed quality documentation. Through staff engagement, targeted improvement areas include better MDT communication and shared responsibility, earlier in-patient emotional/spiritual assessment, developing model LDOLCP examples as resources/benchmark the results are expected to improve. 